\title{
Effect of two training on orexin $A$ and lipid profile in obese adolescent boys
}

\author{
Fezzeh MOHAMADHASANI $^{1}$, Alireza EsFANDYARINEZHAD ${ }^{2, *}$ \\ ${ }^{1,2}$ Msc Exercise Physiology, Teacher of Farzanegan and Shahid Madani High School \\ (Tabriz Sampad) at District 4, Iran \\ *Email: Esfandyarinezhad2009@ Yahoo.Com
}

\begin{abstract}
Given the uncertainties associated with orexin A and obesity, the present study was conducted to identify the effect of endurance training and HIIT on orexin A and lipid Profile in obese adolescent boys. For this purpose, 35 healthy, inactive and obese boys (aged mean 15.5 \pm 0.69 years, BMI percentile equal to or higher than $95 \%$ and BMI $28.72 \pm 2.20 \mathrm{~kg} / \mathrm{m} 2$ ) were divided into three equal groups, control, HIIT and endurance training groups in a semiexperimental, randomized and double-blind design. HIIT and endurance training groups participated high intensity interval and endurance running in eight weeks, three days per week. Orexin A, LDL, HDL, TG, TC Plasma levels and WHR, BF\%, BMI and VO2max were measured before and 48 hours after the last session of training. Data as mean \pm SD using repeated measure ANOVA was analyzed at $\alpha \leq 0.05$. Training was caused significant lower of triglycerides and anthropometric parameters such as body fat percentage, body mass index and waist to hip ratio $(\mathrm{P}<0.05)$. But, significant differences were not observed in orexin $\mathrm{A}$, total cholesterol, LDL and HDL plasma levels, in control and experimental groups $(\mathrm{P}>0.05)$. Therefore, endurance training is more effective than HIIT in improvement of obese adolescent boy's body composition and HIIT is more effective than endurance training to reduce triglycerides. However, changes that may not be in line with changes occurring in orexin A. Thus, it is recommended to repeat like this study in multitimes training with control of sleep, nutrition, puberty changes.
\end{abstract}

Keywords: Orexin A, Endurance training, HIIT (high intensity interval training), Anthropometric parameters, Obesity, Lipid profile. 
Bulletin de la Société Royale des Sciences de Liège, Vol. 85, 2016, p. 653 - 657

\section{Introduction}

Orexin, also called Hypocretin (Hcrt), is a neurotransmitter that regulates arousal, wakefulness, and appetite [1]. Sellayah et al discovered, obesity in orexin knockout mice is a result of inability of brown preadipocytes to differentiate into brown adipose tissue (BAT), which in turn reduces BAT thermogenesis [2]. Bronsky et al found negative correlation between orexin A and age, body height, body weight and BMI [3]. Wu et al's study concluded that training (treadmill locomotion, at a wide range of speeds) does not increase orexin A level beyond baseline, whereas yard play produces a substantial increase in orexin A [4].

Thus, orexin A is affected of obesity, age, dietary restriction, emotional aspects and effects of other hormones such as growth hormone and maybe impact on obesity through changes in basal metabolic. However, so far is not done any study on the effect of training just on orexin A merely and this research aims to study the effects of HIIT and endurance training on orexin A and lipid profile in obese adolescent boys without reducing intake calories.

\section{Methods}

35 healthy, inactive boys, range $14-17$ years, BMI $26-33 \mathrm{~kg} / \mathrm{m}^{2}$, were selected from tabriz district 4 students in a semi-experimental, randomized and double-blind design that their BMI percentile according to the CDC 2000 standard charts was equal to or higher than $95 \%$. HIIT and endurance training groups participated in eight weeks of running, three days per week, (HIIT group: 30 second running with an intensity 90-95\% Heart Rate Target, 4-7 repetition per day and two minutes active rest with 50-55\% Heart Rate Target and endurance training group: 25-40 minutes running per day, with an intensity $65-85 \%$ Heart Rate Target).

Training intensity was determined using AEON stethoscope, made in China, the thickness of subcutaneous fat using Jackson Pollock's men seven points equation, VO2max using Rockport walking and also orexin A plasma level using human orexin A kits by ELISA, product CUSABIO, with marking Human orexin A ELISA kit CSB - E08859h of Japan. Orexin A, LDL, HDL, TG, TC Plasma levels and WHR, BF\%, BMI and VO2max were measured before and 48 hours after the last session of training. Data was analyzed using repeated measure ANOVA, Bonferroni test at $\alpha \leq 0.05$. 
Bulletin de la Société Royale des Sciences de Liège, Vol. 85, 2016, p. 653 - 657

Table 1: The mean, standard deviation and variance analysis of repeated test results before and after the test in control and training groups

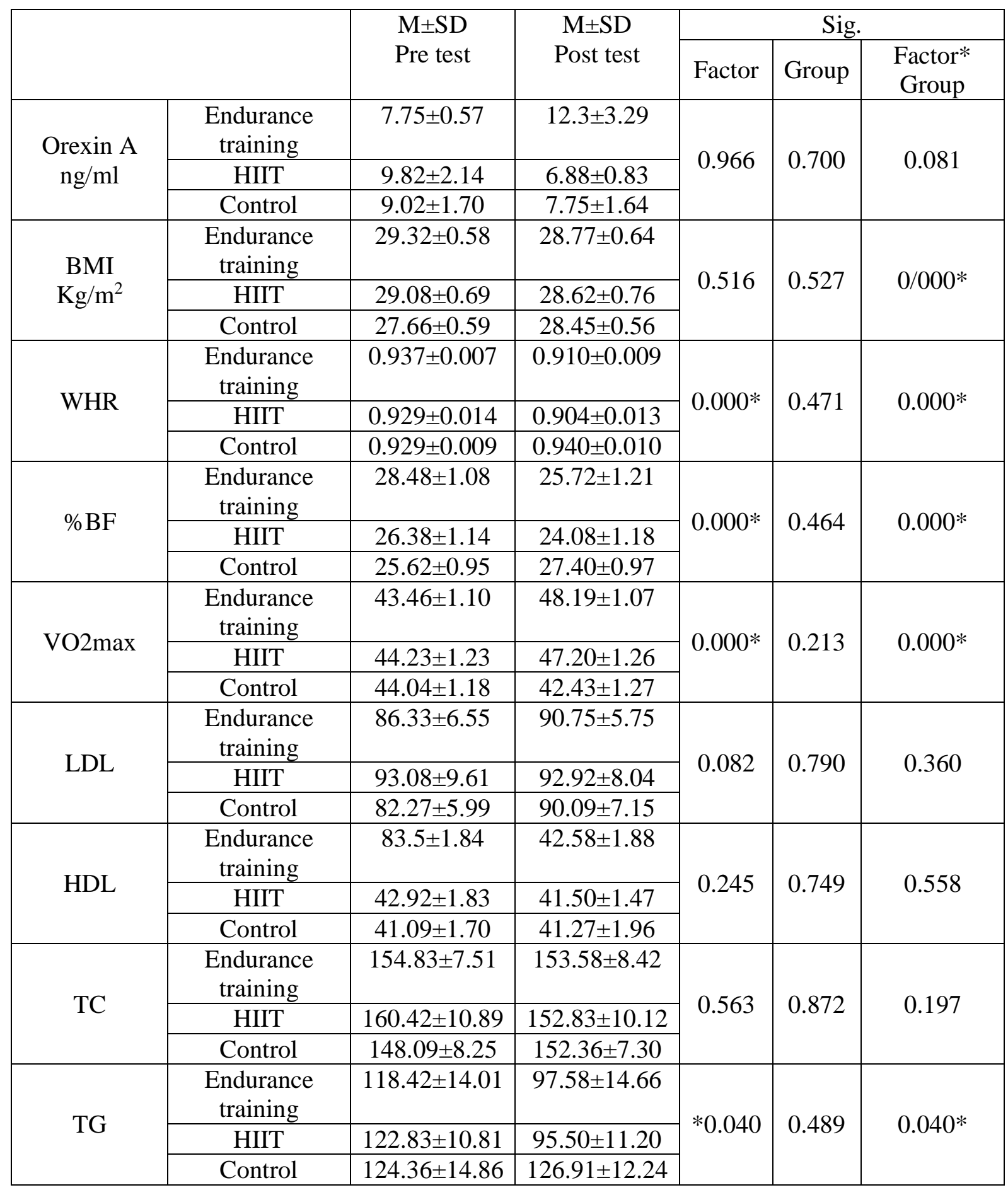




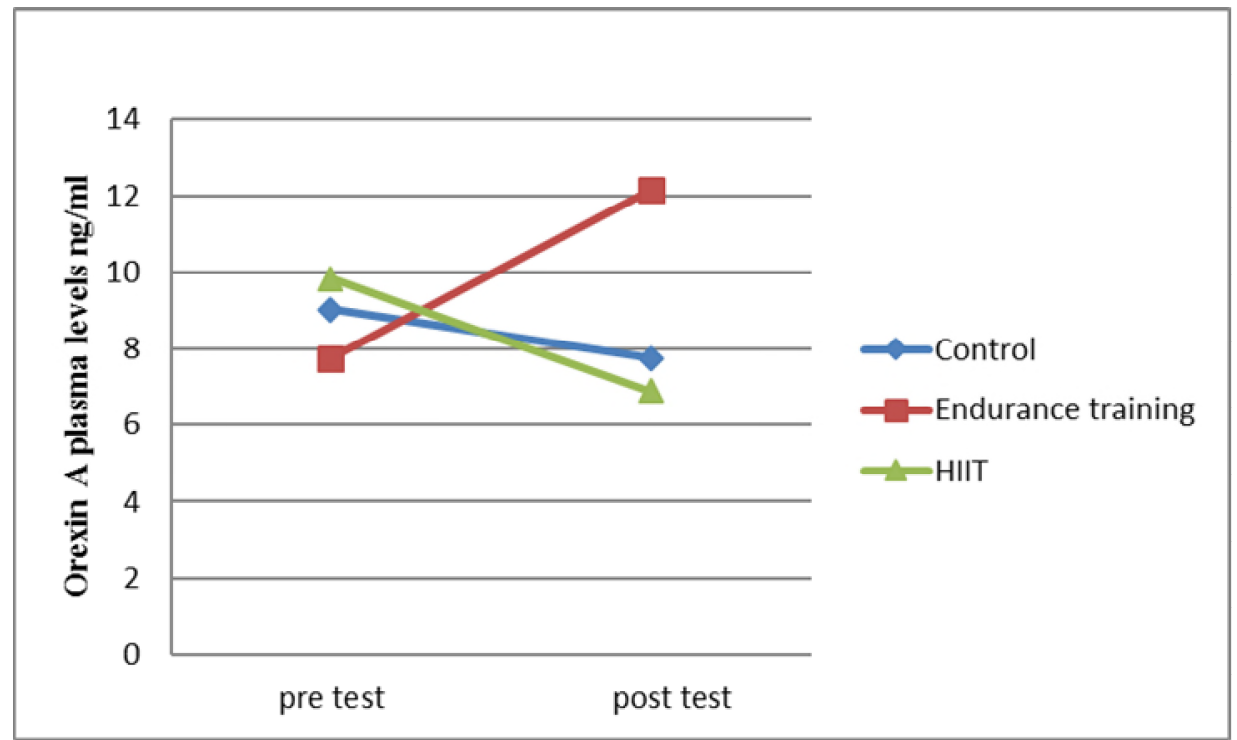

Figure 2: Orexin A plasma levels changes chart after eight weeks of endurance training and HIIT in obese adolescent boys

HIIT and endurance training were caused significant lower of triglycerides and anthropometric parameters such as body fat percentage, body mass index and waist to hip ratio and significant increase of VO2max $(\mathrm{P}<0.05)($ table 1$)$. However, significant differences were not observed at orexin A (figure 2), total cholesterol, LDL and HDL plasma levels in control and experimental groups ( $\mathrm{P}>0.05$ ). According to available Information, the effect of training on orexin-A has been investigated only in Wu et al's study. At their study, in line with current study results, treadmill running did not affectted on cerebrospinalfluid (CSF) Hcrt level in normal dogs[4]. But, contrary to the current study results, Bronsky et al, followed by 5 weeks of physical activity Coupled with dietary restriction found to reverse results, that's mean increase in orexin-A level [3]. Probably, the difference of results of this study with Bronsky's study is concerned with:

- nutrition

- training protocol

in Bronsky's study, training includes running, swimming, indoor and outdoor ball games, aerobics, at least 180 min per day and in the current study, subjects were followed only one steady and specific training protocol. Probably, according to Wu's study results, emotional aspects has increased orexin A in Bronsky's study. Hypocretin neurons are heavily innervated by the amygdala and cholinergic arousal-related basal forebrain. They are activated by anticipation of positive reinforcements, such as food and opiate drugs.

- sleep Duration and sleep time

In Fu et al's study, children's sleep time deprive by one hour increased the level of orexin A $2.409 \mathrm{ng} / \mathrm{ml}$ respectively [5]. 
- age subjects

The subjects in this study lived puberty.

In conclusion, based on the data obtained, it can be said: Endurance training is more effective than HIIT in improvement of obese adolescent boy's body composition and VO2max and HIIT is more effective than endurance training to reduce triglycerides. However, changes that may not be in line with changes occurring in orexin A. Thus, taking into consideration the impact of uncontrollable constraints, such as sleep, nutrition, puberty changes and to achieve more definitive results, it is recommended to repeat like this study, with various sports activities like competitive sports, in variety of different age and sex groups.

\section{References}

[1] Jon, F. Davis, Derrick, L. Choi, and Stephen, C. Benoit, (2011). Orexigenic Hypothalamic Peptides Behavior and Feeding, p. 361-2, in Handbook of Behavior, Food and Nutrition, Victor R. Preedy, Ronald Ross Watson, and Colin R. Martin, eds. Springer.

[2] Sellayah, D. Bharaj, P. Sikder, D., (October 2011). Orexin is required for brown adipose tissue development, differentiation, and function. Cell Metab. 14 (4), p. 478-90.

[3] Bronsky, J. Nedvidkova, J. Zamrazilova, H. Pechova, M. Chada, M. Kotaska, K. Nevoral, J. Prusa, R., (2007). Dynamic Changes of Orexin A and Leptin in Obese Children during Body Weight Reduction, Physiol. Res. 56, p. 89-96.

[4] Wu, M.F. Nienhuis, R. Maidment, N. Lam, H.A. and Siegel, J.M. (2013 January). Cerebrospinal fluid hypocretin (orexin) levels are elevated by play but are not raised by exercise and its associated heart rate, blood pressure, respiration or body temperature changes , Arch Ital Biol. 149(4), p. 492-498 .

[5] Fu, Jf. Zhou, F. Xu, Xq. Zou, Cc. Wang, Cl. Huang, K. Liang, L. (2013). Short Sleep Duration as a Risk Factor for Obesity in Childhood Is Associated with Increased Leptin, Ghrelin, and Orexin Levels, HK J Paediatr (new series). 18, p.152-158 